\title{
Performance study of a Green Light Optimized Speed Advisory (GLOSA) Application Using an Integrated Cooperative ITS Simulation Platform
}

\author{
Konstantinos Katsaros*, Ralf Kernchen*, Mehrdad Dianati*, David Rieck ${ }^{\dagger}$ \\ ${ }^{*}$ Centre for Communications Systems Research (CCSR) \\ Department of Electronic Engineering, University of Surrey \\ Guildford, GU2 7XH, United Kingdom \\ E-mail: \{K.Katsaros, R.Kernchen, M.Dianati\}@ surrey.ac.uk \\ ${ }^{\dagger}$ Fraunhofer Institute for Open Communication Systems \\ Kaiserin-Augusta-Allee 31, 10589 Berlin, Germany \\ E-mail: david.rieck@fokus.fraunhofer.de
}

\begin{abstract}
This paper proposes a Green Light Optimized Speed Advisory (GLOSA) application implementation in a typical reference area, and presents the results of its performance analysis using an integrated cooperative ITS simulation platform. Our interest was to monitor the impacts of GLOSA on fuel and traffic efficiency by introducing metrics for average fuel consumption and average stop time behind a traffic light, respectively. For gathering the results we implemented a traffic scenario defining a single route through an urban area including two traffic lights. The simulations are varied for different penetration rates of GLOSA-equipped vehicles and traffic density. Our results indicate that GLOSA systems could improve fuel consumption and reduce traffic congestion in junctions.
\end{abstract}

\section{Index Terms}

vehicular communications, traffic light advisory, fuel consumption, traffic congestion.

\section{INTRODUCTION}

Advances in wireless communications and in particular vehicular communications have led to the advent of cooperative Intelligent Transportation Systems (ITS) [1], [2]. These systems employ vehicular communication technologies such as $802.11 \mathrm{p}$ [3] to enable deployment of applications that could potentially improve road safety, traffic efficiency, and introduce new entertainment and business applications [4]. Exploitation of ITS for traffic congestion control in urban areas as well as fuel consumption reduction are among the most promising applications according to transportation authorities [5], [6]. This can be achieved by vehicle-to-vehicle (V2V) or infrastructureto-vehicle (I2V) communications, intelligently advising individual drivers about traffic events, such as the traffic light phases and beyond.

The potential of V2V communication for improving fuel efficiency has been demonstrated in [7], showing that vehicular communication can assist to reduce average fuel consumption especially under high traffic density and long traffic light cycles. Other projects have investigated the impacts on fuel efficiency when using wireless communications between vehicles (V2V) or vehicle-to-infrastructure (V2I) employing different algorithms to smoothly slow down at a red traffic light or to reach it at the next green phase. Depending on the used consumption models, different results can be observed. One important aspect, as introduced in [8], is the dependency of the results on different penetration rates of the communication enabled vehicles. In the same work as well as in [9], the effect of traffic density is studied. In [8] it is also suggested to either cut the fuel delivery or stop the engine in order to achieve less fuel consumption. In [10], the IDM car following model [11] is used to control a platoon of 10 vehicles, where only the leading one is equipped with communication capabilities, achieving $30 \%$ fuel savings. In [12] when the algorithm is used only with one vehicle and one traffic light, fuel savings reached 20\%. Although these results seem trustworthy, the simulations are conducted with small number of vehicles which does not consider the dynamics of the vehicular environment. When the model in [12] is scaled up including 15 traffic light junctions, 
the results are reduced to $8 \%$ reduction in fuel consumption. The optimal activation distance for the algorithm is also investigated in this work and is found that a distance of $500 \mathrm{~m}$ achieves the best results in their simulations.

In [10] and [9] traffic efficiency is examined using different performance metrics. In [10], the increase of average speed is taken as indicator of traffic efficiency where in [9], the flow and the ratio of motionless vehicles are considered. We examine the average stop time behind a traffic light as a metric of traffic efficiency. This is because we want to see not only how many vehicles are stopped like [9] but also how long they wait.

In the aforementioned works, different communication technologies are adopted. The authors in [7], [9], [12], [13] do not discuss in depth the communication mechanisms of their simulations. They assume successful dissemination of the messages. Others like [10] and [14] use general purpose wireless communication technologies, such as wireless sensor networks and IEEE802.11 [15]. Lately the IEEE 802.11 standard is preferred due to operational cost. The highly dynamic network topology of vehicular communications has led to the introduction of IEEE 802.11p [3] that is more suitable for such applications and is the one we used in our simulations.

This paper aims to implement a GLOSA system to reduce traffic congestion by decreasing the average stop time behind traffic lights while reducing fuel consumption and $\mathrm{CO}_{2}$ emissions. The GLOSA application provides the advantage of timely and accurate information about traffic lights cycles and traffic lights position information through infrastructure-to-vehicle (I2V) communication, and provides drivers with speed advice guiding them with a more constant speed and with less stopping time through traffic lights. The main challenges in achieving this include the modelling of the vehicle traffic, the communications between traffic lights and vehicles and finally the driver's behaviour. Individual research has been performed for each one of these areas, but complete simulations by taking into account the dynamics of all parameters are scarce. Thus, for our implementation of GLOSA, we used an integrated simulation tool based on the Fraunhofer VSimRTI [16], which enables online two-way coupling of different simulators for monitoring the influence of GLOSA application on the traffic and fuel consumption. Our results show up to $7 \%$ reduction in average fuel consumption and up to $89 \%$ in average stop time. While conducting the simulations we found that an optimal GLOSA activation distance is around $300 \mathrm{~m}$.

The rest of this paper is organised as follows. In section II, we present our integrated simulation approach, and in section III, we give an overview of the integrated simulation platform. The design of the GLOSA algorithm is thoroughly discussed in section IV. In section V, the simulation set-up is presented followed by our evaluation results. Finally in section VI we conclude and provide ideas for future work.

\section{Simulation APPROACH}

We designed and tested GLOSA in an integrated simulation platform. The first step was to define a reference scenario which is depicted in Fig. 1. In this reference area we placed a traffic light. Vehicles will enter following a common arrival process such as Poisson distribution. In the scenario the equipped vehicles rate is varied, since we want to investigate the influence of the penetration rate and which is the minimum percentage of equipped vehicles for GLOSA to have a positive impact on the traffic efficiency and fuel consumption. Road traffic will be modelled using the microscopic Stefan Krauss (SK) model [17], a car-following model with two basic rules. First, vehicles in free motion have a target speed and try to cruise at it. Second, when a vehicle senses the distance to the vehicle ahead to be less than a certain threshold, it slows down keeping a safe distance. The speed of the vehicles is within a certain range $\left[V_{\min }, V_{\max }\right]$ where $V_{\min }$ is the minimum speed that vehicles can cruise without causing further traffic congestion and $V_{\max }$ is the maximum speed that is forced by the speed limit of the area. Acceleration is also bounded and asymmetric - higher deceleration than acceleration - for more realistic simulations. The SK model is integrated in SUMO [18] traffic simulator that we used in our work.

We have set up two simulation scenarios in order to compare the results. In the first scenario $\left(S_{0}\right)$, there is no driver's assistance information and the traffic is governed by the SK model. In the second scenario $\left(S_{1}\right)$ we provide information through the GLOSA messages and the driver receives speed advisory messages. We assume that all drivers who get a speed advise, will follow it. We control the percentage of GLOSA equipped vehicles to monitor the impact of penetration rate. Therefore, we have defined two performance metrics that we check in all scenarios in order to derive our conclusions. The first metric $\left(P_{1}\right)$ is the average stop time of vehicles waiting at the intersection behind red lights measuring the traffic efficiency of GLOSA application. We assume that there is no other reason for the vehicles to halt apart from stopping at a traffic light or a queue caused by the traffic light (we do not simulate brake downs or accidents in our scenarios). The second metric $\left(P_{2}\right)$ is the fuel consumption derived 


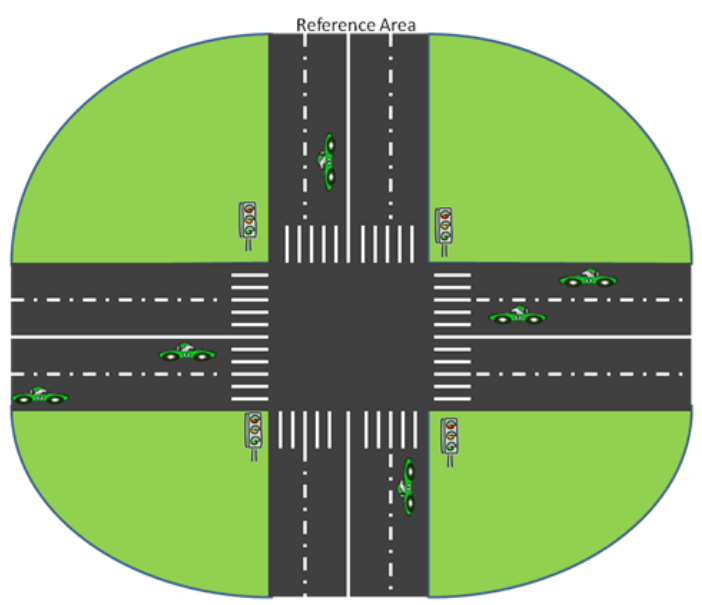

Fig. 1. Reference Scenario

from the fuel consumption and emission model in [19] measuring the fuel efficiency of GLOSA application. The emissions of $\mathrm{CO}_{2}$ are estimated from the same model to be proportional to fuel consumption.

\section{InTEgRATED Simulation Platform}

Simulating the GLOSA use case poses a challenge in terms of combining and synchronizing different simulation aspects, e.g. vehicular traffic, network communication and application handling. To address these challenges, the integrated simulation platform VSimRTI was used to simulate this use case. VSimRTI borrows some concepts of the High Level Architecture (HLA) [20] to enable the coupling of the most appropriate simulators for a scenario. VSimRTI is a lightweight framework, which is responsible for tasks such as synchronization of simulators, interaction between them and lifecycle management of simulators but also applications. VSimRTI supports V2Xspecific elements such as road-side units or application-equipped vehicles from the beginning.

To simulate the GLOSA application, VSimRTI_app was used. VSimRTI_app is an application simulator developed for VSimRTI. It provides a couple of simple JAVA interfaces to create V2X applications, while offering access to all relevant simulation data such as vehicle status or communication modules. Messages sent to specific vehicles are forwarded to the associated application and application output can be directed to a specific vehicle (e.g. giving speed advisory to a vehicle).

\section{GLOSA ALgORITHM}

The GLOSA algorithm has been implemented to support the aforementioned simulation approach and is presented in the Algorithm 1. First, vehicles enter the communication range of a traffic light according to the Poisson distribution as mentioned before. The RSU (Road Side Unit) attached to a traffic light broadcasts periodically CAM's (Co-operative Awareness Messages) including the position, timing information and additional data for the traffic light. When OBU receives a CAM, the algorithm checks if its source is a traffic light or not. From the position information within the message and the vehicle's own position and heading, it calculates whether this traffic light is relevant (on its route) or not (line 1). The application can then calculate the distance from the traffic light and with the current speed and acceleration, the time that it would take to reach it (Time-to-Traffic-Light $T_{T L}$ ) (line 2). Next, it checks the traffic light phase at that time $\left(T_{T L}\right)$ (line 3). If the traffic light is green when the vehicle reaches it, then the vehicle continues its trip trying to reach the maximum speed limit of the road (lines 4-6). If it is red, it calculates the speed that it should have in order to reach it in the next green phase (lines 7-9). If it is yellow, depending on the remaining yellow time and the acceleration capabilities of the vehicle it could advice to accelerate or decelerate again within the permitted range (lines 10-13). Finally the driver gets an advise with the speed limited within the permitted range $\left[V_{\min }, V_{\max }\right]($ line 15). This algorithm runs every second which makes it more robust against external interference, such as other vehicles, that do not follow the same advisory speed or are non-equipped. 


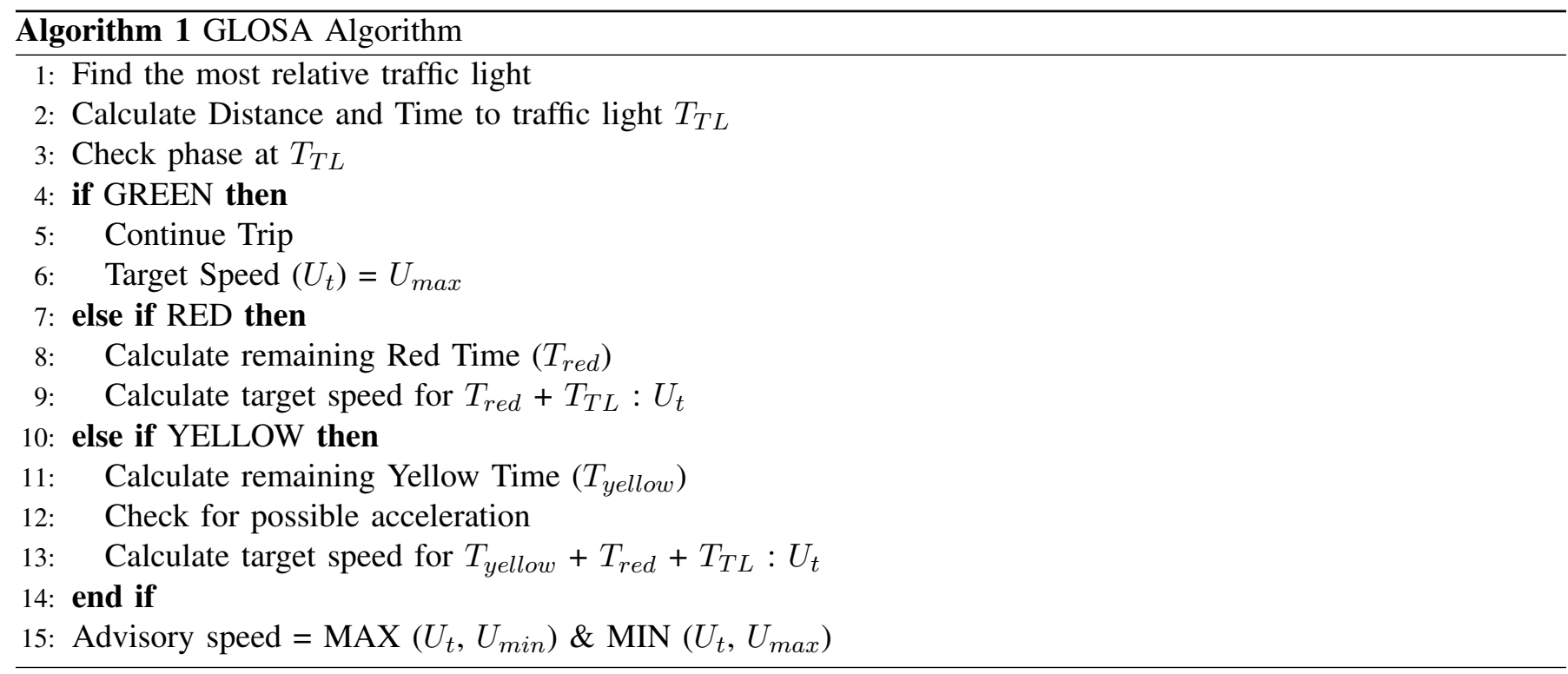

The algorithm gets as input the current speed $U_{0}$, acceleration $a$ of the vehicle and the distance to the traffic light $D_{t l}$. Using basic rules of motion, given by (1)

$$
d=u * t+1 / 2 * a * t^{2}
$$

where $d$ is the distance, $u$ is the initial speed, $t$ is the time and $a$ is the acceleration, the time to reach the traffic light $\left(T_{T L}\right)$ can be calculated as shown in (2).

$$
T_{T L}= \begin{cases}\frac{d}{u} & \text { when } \mathrm{a}=0 \\ -\frac{u}{a}+\sqrt{\frac{u^{2}}{a}+\frac{2 d}{a}} & \text { when } \mathrm{a} \neq 0\end{cases}
$$

The target speed $\left(U_{t}\right)$ for the red and yellow light phase is calculated using (3)

$$
U_{t}=\frac{2 * d}{t}-U_{0}
$$

where $d$ is the distance to traffic light $\left(D_{t l}\right), t$ is the time to reach the traffic $T_{T L}$ light plus the remaining time for the next green phase ( $T_{\text {red }}$ or $T_{\text {yellow }}+T_{\text {red }}$ respectively) and $U_{0}$ is the current speed.

\section{Simulation SET-UP AND EVALUATION RESUltS}

For the evaluation of the GLOSA application a series of simulations were conducted. The configuration of the environment for the project consists of the SUMO [18] traffic simulator used to produce and cope with the vehicle traffic, the JiST/SWANS [21] is used for the communications and finally an application simulator which runs the GLOSA application written in Java. The underlying simulation scenario is a road network section of Guildford town centre in United Kingdom as depicted in Fig. 2. Vehicles start from point A on York Road according to a Poisson distribution and travel until point B on Waterden Road on one lane and without overtaking. The number of vehicles is defined to 100 in order to gather sufficient data in terms of time and number of independent vehicles to get statistically accurate results. The travel distance is 0.6 miles $(0.965 \mathrm{~km})$. Within this route there are two traffic lights $\left(T L_{1}\right.$ and $T L_{2}$ ). For these two traffic lights the timing regarding the previous route is: 20-4-6 (Green-Yellow-Red) and 20-4-36 seconds, respectively. The difference in red time for $T L_{2}$ is because of the London Road's green phase duration. The speed limit on the road is set to $15 \mathrm{~m} / \mathrm{s}(54 \mathrm{~km} / \mathrm{h})$ which is near the usual limit in an urban area. The minimum advisory speed is set to $6 \mathrm{~m} / \mathrm{s}(21.6 \mathrm{~km} / \mathrm{h})$ in order not to travel to slow and cause more congestion. The simulation runs until every vehicle has travelled the whole distance from point A to point $\mathrm{B}$. The communication range of the traffic lights is set near 500m and using IEEE $802.11 \mathrm{p}$ communication as access mechanism they broadcast their CAM's. 


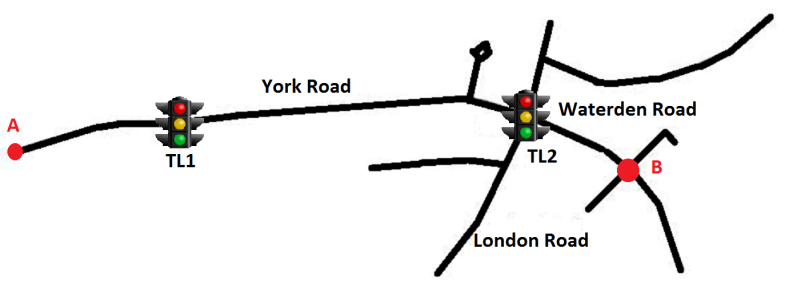

Fig. 2. Simulation Scenario Map

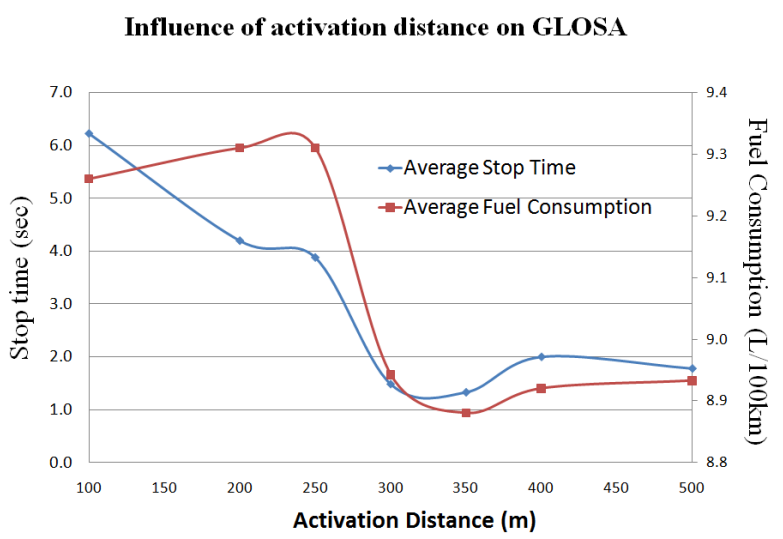

Fig. 3. Influence of activation distance on GLOSA performance

The simulations can be divided into three categories. First, we tested the influence of the activation distance for GLOSA on the overall performance. In these simulations all vehicles are equipped with the GLOSA application. Also, in order to check the integrity of the algorithm we excluded the first traffic light and run only with one $\left(T L_{2}\right)$. The timing for this was also altered in order to have equal red and green phases. The results of the two performance metrics can be seen in Fig. 3. An optimal point of activation is found at a distance near $350 \mathrm{~m}$. At shorter activation distances, the reaction time (time required for the driver to slow down to the advised velocity) is not enough to have benefits. The fuel consumption is also slightly increased due to the fact that the average trip time is increased (vehicles are advised to travel at lower speeds). At longer activation distances, the benefits regarding fuel consumption are slightly decreased but remain near the optimal levels. The results for two traffic lights with a distance near $400 \mathrm{~m}$ between them (Fig. 2), shift this optimal point to a shorter distance of $250 \mathrm{~m}$ which will be the value used to produce the next set of results. This is due to the fact that vehicles do not have enough time to accelerate and reach a higher velocity after the effect the first traffic light has on their velocity before they run the GLOSA algorithm once again for the second traffic light. Hence, further simulations have to be made for larger scale scenario and more traffic lights to conclude which activation distance to use. Having a shorter activation distance means that we can reduce the transmission power of the RSU and thus having better resource allocation and less collisions in the communications. Compared with the work in [12], where the minimum activation distance is found near $500 \mathrm{~m}$, our work shows better characteristics in this aspect.

Second, we measured the influence that GLOSA penetration rate has on the two performance metrics and how the non-equipped vehicles are affected. The simulations were conducted in a high traffic density environment (Poisson expected value $\lambda=0.2$ ). From [8], we learn that an increase in penetration rates of equipped vehicles allows for a better reduction of fuel consumption in the overall traffic scenarios. As it can be seen from Fig. 4 and Fig. 5 this is verified not only for fuel efficiency, but also for traffic efficiency. The most interesting outcome from these figures is that even the non-equipped vehicles are getting affected in a beneficial way from the GLOSA equipped vehicles and this is due to the SK model. They follow the leading vehicle which - if equipped with GLOSA - forces them to adjust their speeds accordingly since we assume that there are no overtaking in our simulations. The second notice is that the average stop time is reduced even when the penetration rate is small, but in order to see positive results in fuel efficiency we need at least $50 \%$ equipped vehicles. The observed average maximum reduction in fuel consumption is $7 \%$ which is slightly higher than the average maximum fuel savings in [12] for their scaled 


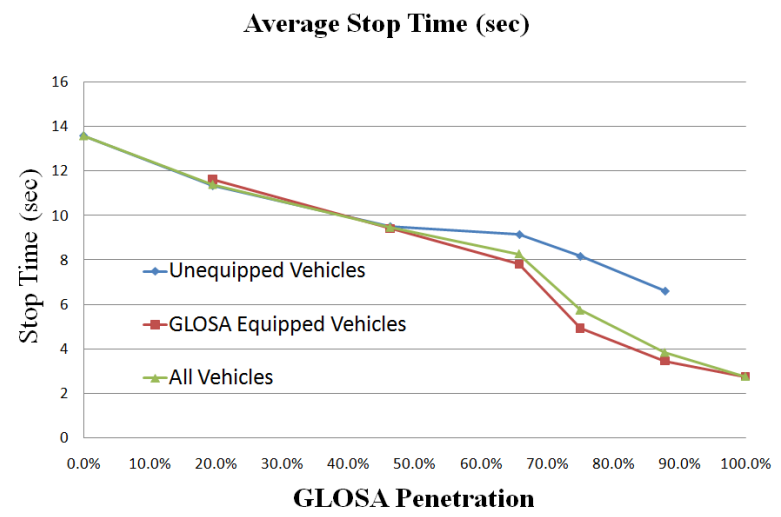

Fig. 4. Influence of GLOSA penetration rate on average stop time

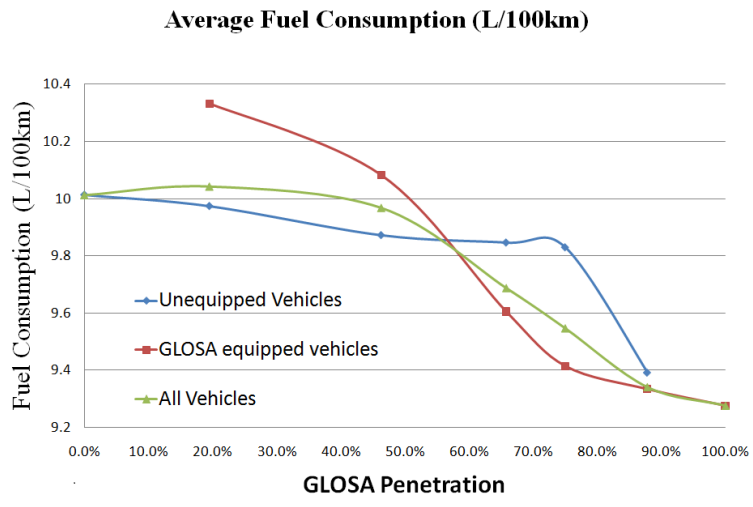

Fig. 5. Influence of GLOSA penetration rate on average fuel consumption

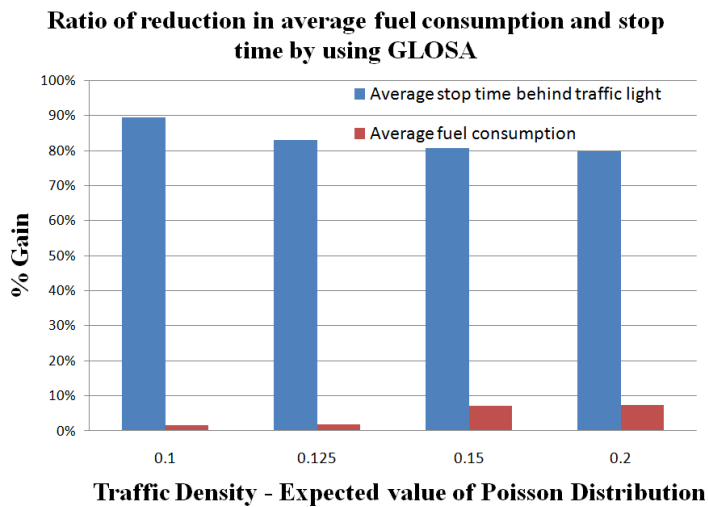

Fig. 6. Influence of vehicle traffic density on the GLOSA performance

up scenario.

Finally, we simulated different traffic densities (high, medium and low) in order to capture the influence they have on the overall performance of the GLOSA application. The results shown in Fig. 6 suggest that the higher the traffic density (moving from left to right in the plot), the more benefits we have regarding in fuel efficiency reaching a maximum of $7 \%$ fuel reduction. On the other hand, the benefits we get regarding traffic efficiency are decreased, which was also reported in [9]. This is because the vehicles are more scarcely distributed therefore they do not influence each other, they all follow precisely the advisory speed and there are smaller queues at the traffic lights making the GLOSA algorithm work better. 


\section{CONCLUSIONS AND FUTURE WORK}

The results suggest that the GLOSA application has a positive effect on both performance metrics. The higher the GLOSA penetration rate is, the more benefits we have with a maximum of $80 \%$ reduction in stop time and up to $7 \%$ reduction in fuel consumption in a high traffic density scenario. There is a critical point of $50 \%$ of equipped vehicles where the effect of GLOSA starts to be more visible on fuel consumption. As the density decreases, the benefits for fuel efficiency are reduced, but there is still improvement compared to non-equipped vehicles. The traffic efficiency on the other hand is increased with the decrease in traffic density reaching $89 \%$. There is also an optimal activation distance where the GLOSA application should advise the driver and this is near 300m from the traffic lights but it depends slightly on the road network. Closer to this distance, the time to react is limited and further away there are no more benefits. If the complexity of the algorithm is to be increased, the distance could be also increased. The work presented by this paper is an example of what can be achieved in terms of fuel and traffic efficiency when vehicles are enabled to communicate with traffic lights and how we can exploit an integrated simulation platform to achieve this.

There are various ways in which this application could be extended to achieve more accurate results. First of all, we assumed that there are no vehicles waiting at the traffic light which is not always the case. Therefore, the distance to traffic light could be replaced by the distance to the end of the queue instead to achieve more reasonable results. The simulation network should also be extended to a larger scale scenario using real data for vehicle input. Finally having results from field tests would provide data to compare field tests and simulations to evaluate the estimations made by the simulations.

\section{ACKNOWLEDGMENT}

The work was done within the joint research project PREDRIVE C2X, which is funded by DG Infso of the European Commission within the 7th Framework Program.

\section{REFERENCES}

[1] L. Figueiredo, I. Jesus, J. Machado, J. Ferreira, and J. Martins de Carvalho, "Towards the development of intelligent transportation systems," 2001, pp. $1206-1211$.

[2] H. Hartenstein and K. P. Laberteaux, "A tutorial survey on vehicular ad hoc networks," IEEE COMMUNICATIONS MAGAZINE, vol. 46, no. 6, pp. 164-171, JUN 2008, aCM MobiCom/MobiHoc 2007, Montreal, CANADA, 2007.

[3] "Draft standard for information technology-telecommunications and information exchange between systems-local and metropolitan area networks -specific requirements part 11: Wireless lan medium access control (mac) and physical layer (phy) specifications amendment 7: Wireless access in vehicular environments," IEEE Unapproved Draft Std P802.11p/D9.0, July 2009, 2009.

[4] "Car 2 car communication consortium manifesto," Online, CAR 2 CAR Communication Consortium, www.car-2-car.org.

[5] "National traffic signal report card," Online, National Transportation Operations Coalition, 2007, http://www.ite.org/REPORTCARD.

[6] "Traffic congestion and urban mobility," Online, Texas Transportation Institute, 2010, http://tti.tamu.edu/infofor/media/topics/congestion mobility.htm.

[7] A. Widodo, T. Hasegawa, and S. Tsugawa, "Vehicle fuel consumption and emission estimation in environment-adaptive driving with or without inter-vehicle communications," in PROCEEDINGS OF THE IEEE INTELLIGENT VEHICLES SYMPOSIUM 2000, 2000, Proceedings Paper, pp. 382-386.

[8] A. Wegener, H. Hellbruck, C. Wewetzer, and A. Lubke, "Vanet simulation environment with feedback loop and its application to traffic light assistance," nov. 2008, pp. $1-7$.

[9] M. Chao-Qun, H. Hai-Jun, and T. Tie-Qia, "Improving urban traffic by velocity guidance," in INTERNATIONAL CONFERENCE ON INTELLIGENT COMPUTATION TECHNOLOGY AND AUTOMATION, VOL 2, PROCEEDINGS, 2008, Proceedings Paper, pp. 383-387.

[10] M. Sanchez, J.-C. Cano, and D. Kim, "Predicting traffic lights to improve urban traffic fuel consumption," in 2006 6th International Conference on ITS Telecommunications Proceedings. IEEE, 2006, Proceedings Paper, pp. 331-336.

[11] M. Treiber, A. Hennecke, and D. Helbing, "Congested traffic states in empirical observations and microscopic simulations," PHYSICAL REVIEW E, vol. 62, no. 2, Part A, pp. 1805-1824, AUG 2000.

[12] T. Tielert, K. M., H. Hartenstein, R. Luz, H. S., and T. Benz, "The impct of traffic-light-to-vehicle communication on fuel consumption and emmisions," in Internet of Things 2010 - Second International Conference for Academia and Industry, 2010.

[13] B. Asadi and A. Vahidi, "Predictive cruise control: Utilizing upcoming traffic signal information for improving fuel economy and reducing trip time," Control Systems Technology, IEEE Transactions on, vol. PP, no. 99, pp. 1-9, 2010.

[14] I. Iglesias, L. Isasi, M. Larburu, V. Martinez, and B. Molinete, "I2v communication driving assistance system: On-board traffic light assistant," sep. 2008, pp. $1-5$.

[15] "Supplement to ieee standard for information technology - telecommunications and information exchange between systems - local and metropolitan area networks - specific requirements. part 11: Wireless lan medium access control (mac) and physical layer (phy) specifications: High-speed physical layer in the 5 ghz band," IEEE Std 802.11a-1999, p. i, 1999. 
[16] T. Queck, B. Schünemann, I. Radusch, and C. Meinel, "Realistic simulation of v2x communication scenarios," in APSCC '08: Proceedings of the 2008 IEEE Asia-Pacific Services Computing Conference. Washington, DC, USA: IEEE Computer Society, 2008, pp. 1623-1627.

[17] S. Krauss, "Microscopic modeling of traffic flow: Investigation of collision free vehicle dynamics," Ph.D. dissertation, 1998.

[18] D. Krajzewicz, G. Hertkorn, C. Rssel, and P. Wagner, "Sumo (simulation of urban mobility) - an open-source traffic simulation," sep. 2002, pp. 183-187.

[19] R. D.C.Biggs, "An energy-related model of instantaneous fuel consumption," Traffic engineering \& control, vol. 27, pp. 320-325, 1986.

[20] Institute of Electrical and Electronics Engineers, IEEE standard for modeling and simulation (M\&S) High Level Architecture (HLA)federate interface specification. IEEE Standard 1516.1. New York, NY, USA: IEEE, 2000.

[21] R. Barr, Z. J. Haas, and R. van Renesse, "Jist: an efficient approach to simulation using virtual machines:research articles," Softw. Pract. Exper., vol. 35, no. 6, pp. 539-576, 2005. 